\title{
Increase in the astaxanthin synthase gene (crtS) dose by in vivo DNA fragment assembly in Xanthophyllomyces dendrorhous
}

\author{
Gabriela Contreras ${ }^{1}$, Salvador Barahona ${ }^{1}$, María Cecilia Rojas², Marcelo Baeza ${ }^{1}$, Víctor Cifuentes ${ }^{1}$ \\ and Jennifer Alcaíno ${ }^{1 *}$
}

\begin{abstract}
Background: Xanthophyllomyces dendrorhous is a basidiomycetous yeast that is relevant to biotechnology, as it can synthesize the carotenoid astaxanthin. However, the astaxanthin levels produced by wild-type strains are low. Although different approaches for promoting increased astaxanthin production have been attempted, no commercially competitive results have been obtained thus far. A promising alternative to facilitate the production of carotenoids in this yeast involves the use of genetic modification. However, a major limitation is the few available molecular tools to manipulate $X$. dendrorhous.

Results: In this work, the DNA assembler methodology that was previously described in Saccharomyces cerevisiae was successfully applied to assemble DNA fragments in vivo and integrate these fragments into the genome of $X$. dendrorhous by homologous recombination in only one transformation event. Using this method, the gene encoding astaxanthin synthase (crtS) was overexpressed in X. dendrorhous and a higher level of astaxanthin was produced.
\end{abstract}

Conclusions: This methodology could be used to easily and rapidly overexpress individual genes or combinations of genes simultaneously in $X$. dendrorhous, eliminating numerous steps involved in conventional cloning methods.

Keywords: Xanthophyllomyces dendrorhous, Astaxanthin synthase, DNA assembler

\section{Background}

Xanthophyllomyces dendrorhous is a basidiomycetous yeast with potential in the biotechnology industry as it is able to synthesize carotenoids, particularly astaxanthin. Carotenoids are natural yellow, orange, or red pigments, and more than 700 different carotenoid chemical structures have been described to date [1]. Animals are unable to synthesize these pigments de novo and can only obtain them in their diet. These pigments are currently used as food colorants and have received attention for their ability to alleviate chronic diseases due to their antioxidant properties, which can mitigate the damaging effects of oxidative stress induced by reactive oxygen species (ROS) [2]. Among carotenoids, astaxanthin (3,3'-dihydroxy- $\beta$,

\footnotetext{
* Correspondence: jalcainog@u.uchile.cl

'Departamento de Ciencias Ecológicas, Facultad de Ciencias, Universidad de Chile, Las Palmeras 3425, Casilla, Santiago 653, Chile

Full list of author information is available at the end of the article
}

$\beta$-carotene-4-4'-dione) is notable based on its antioxidant properties, which are greater than those of beta-carotene or even alpha-tocopherol [3], and the application of astaxanthin in the pharmaceutical and cosmetic industries has recently been explored [4]. Astaxanthin has also been widely used in the aquaculture industry as a colorant for cultured salmonids to achieve the flesh color that is preferred by consumers. In addition, astaxanthin is an essential nutritional component for proper fish growth and reproduction, making this compound a significant factor in aquaculture production costs [4].

$X$. dendrorhous is one of the few microorganisms that produces astaxanthin. However, the production of this pigment by wild-type strains is too low (200-400 $\mu \mathrm{g}$ per g of dry yeast) to provide a natural source that is economically competitive with chemical synthesis of this pigment. Therefore, many efforts have attempted to improve the astaxanthin production from $X$. dendrorhous,

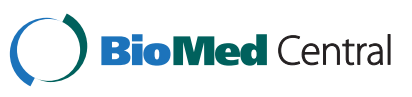


including optimization of culture conditions such as glucose concentration [5,6], oxygen levels [6,7], $\mathrm{pH}[8,9]$, carbon/nitrogen ratio [10], and light intensities [11], in addition to classic random mutagenesis methods [12-15]. A promising alternative to increase the astaxanthin yield in this yeast is to overexpress the genes involved in carotenoid synthesis (Figure 1) for which several attempts have been performed (For a review see: [16]). A small increase in the amount of total carotenoids was obtained by overexpressing the gene encoding phytoene synthase-lycopene cyclase $(\operatorname{crt} Y B)$ by integrating multiple copies of this gene into the ribosomal DNA [17]. However, this increase was mainly due to increased synthesis of beta-carotene and echinenone, while the astaxanthin content was slightly reduced (or unaffected). In another experiment in which the gene encoding phytoene desaturase ( $c r t I)$ was overexpressed, the overall carotenoid production decreased including a 50\% reduction in the astaxanthin fraction [17]. Similarly, the overexpression of the gene encoding isopentenyl pyrophosphate isomerase (idi) led to a decrease in the amount of total carotenoids [18]. In another work attempting to promote metabolite flow towards carotenoid biosynthesis, the cDNA encoding the geranylgeranyl pyrophosphate synthase gene (crtE) was overexpressed, resulting in a strain with slightly higher carotenoid levels, although the astaxanthin levels were not increased [19]. However, the simultaneous insertion of extra copies of the $\operatorname{crt} Y B$ and crtS (which encodes the astaxanthin synthase) genes in an astaxanthin-overproducing strain obtained by random mutagenesis resulted in transformants with an even higher astaxanthin content [20]. Therefore, the overexpression of a single gene is not enough to significantly increase the astaxanthin level in $X$. dendrorhous, and overexpression of

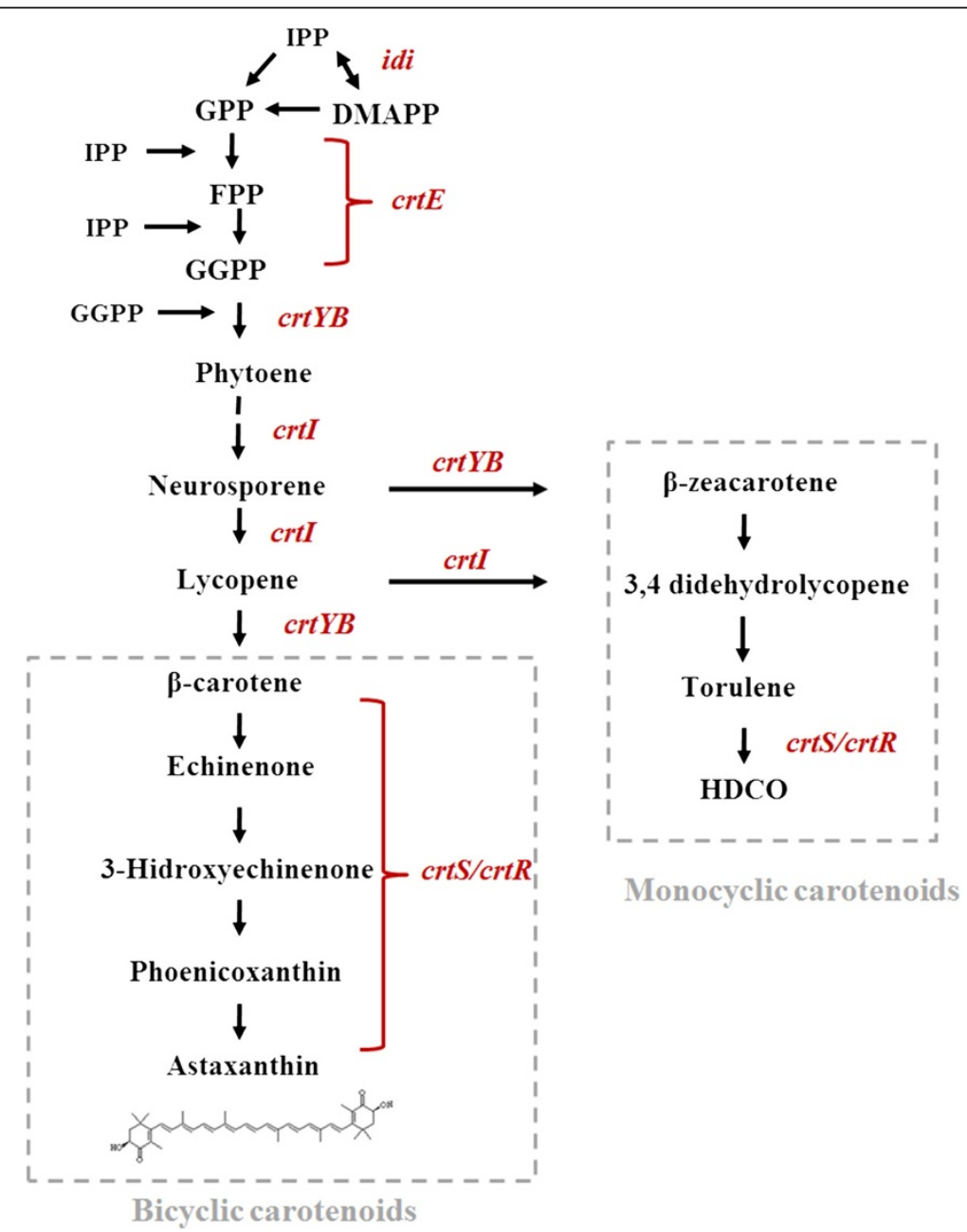

Figure 1 Diagram of the astaxanthin biosynthetic pathway in $\boldsymbol{X}$. dendrorhous. The astaxanthin biosynthesis pathway in $X$. dendrorhous proposed by [21] and [22]. Metabolite abbreviations: IPP (isopentenyl pyrophosphate), DMAPP (dimethylallyl pyrophosphate), GPP (geranyl pyrophosphate), FPP (farnesyl pyrophosphate), GGPP (geranylgeranyl pyrophosphate) and HDCO (3-hydroxy-3',4'-didehydro- $\beta, \psi$-carotene-4-one). The names of the genes controlling each step are written in red: idi (IPP-isomerase), crtE (geranylgeranyl pyrophosphate synthase), crtYB (PBS, phytoene- $\beta$-carotene synthase), crtl (PDS, phytoene desaturase), crtS (astaxanthin synthase) and crtR (cytochrome P450 reductase). 
different combinations of carotenogenic genes seemed to provide better results.

One of the major limitations in the genetic manipulation of $X$. dendrorhous is the limited number of molecular tools available to transform and engineer this yeast. Recently, an in vivo method for DNA fragment assembly and yeast transformation, DNA assembler, was reported [23]. With this technique, numerous DNA fragments are assembled in vivo via homologous recombination at their ends following a single transformation event, allowing the incorporation of an entire heterologous biochemical pathway in Saccharomyces cerevisiae. As the $X$. dendrorhous homologous recombination machinery has previously been successfully exploited for the development of transformation strategies and gene function analysis [17,24-27], in this work, we adapted the DNA assembler methodology to overexpress the gene encoding astaxanthin synthase ( $c r t S)$ that catalyzes the formation of astaxanthin from beta-carotene $[28,29]$ in $X$. dendrorhous.

\section{Results \\ In vivo assembly and integration of DNA fragments into the genome of $X$. dendrorhous}

To evaluate the feasibility of assembling DNA fragments in vivo in $X$. dendrorhous, a DNA cassette containing only the gene that confers resistance to hygromycin $\mathrm{B}$ [30] was integrated in the genome of the wild-type $X$. dendrorhous strain UCD 67-385. A 1,260 bp locus called DHS3 [GenBank: JN835289.1] was chosen as one of the potential target site, which is located at 2,110 bp downstream of the $X$. dendrorhous HIS3 gene. This region is transcribed and encodes an uncharacterized gene product, so we expected that its interruption would not drastically affect the physiology of the yeast.

Three DNA fragments (454 bp DHS3 "up" for upstream, 1,817 bp hygromycin B resistance cassette and 460 bp DHS3 "down" for downstream) were prepared as illustrated in Figure 2A. First, each fragment was individually PCR amplified with a set of primers designed to make the $5^{\prime}$ end of the fragment overlap the $3^{\prime}$ end of the preceding DNA fragment. In this way, the hygromycin $B$ resistance cassette overlaps the two flanking DNA fragments targeting the DHS3 locus. The overlapping region contained approximately $100 \mathrm{bp}$ of sequence homology between fragments to allow in vivo homologous recombination between them. A diploid $X$. dendrorhous wild-type strain [31] was co-transformed with the three DNA fragments by electroporation. Assembly of these fragments and their integration into the genome was accomplished, as nine hygromycin B-resistant transformants were obtained. PCR analyses confirmed that all of them contained the resistance cassette at the expected integration target. However, as the starting strain UCD $67-385$ is diploid [31], a wildtype DHS3 allele was still detected in the resulting transformants, indicating that they were heterozygous at this locus. For this reason, one of the transformants was randomly chosen (named $X d_{-} 1 \mathrm{H}$, for one hygromycinresistance cassette copy) to obtain the homozygous strain $X d \_2 \mathrm{H}$ ( $2 \mathrm{H}$ for two copies of the hygromycin-resistance cassette) using the double recombinant method (DRM) [30] (Figure 2A). Panel B of Figure 2 shows the amplicons obtained from genomic PCR confirming the integration of the hygromycin $\mathrm{B}$ resistance cassette into the expected locus in strains $X d \_1 \mathrm{H}\left(D H S 3 / d h s 3^{:: h p h}\right)$ and $X d \_2 \mathrm{H}$ $\left(d h s 3^{:: h p h} / d h s 3^{:: h p h}\right)$. To the naked eye, the color of the heterozygous and homozygous strains is identical to the wildtype strain and both strains are able to grow in minimal medium (Figure 2C). Total carotenoids were quantified and no significant differences were detected between the transformants and the wild-type strain (data not shown). Thus, the interruption of the DHS3 locus did not cause auxotrophy or greatly affected the carotenogenesis in this yeast.

To confirm that this transformation methodology is effective for other integration targets, the resistance cassette was successfully integrated into two other genomic loci. However, the color phenotype of the transformants was different from the parental strain (data not shown).

\section{Increase of crts gene dose}

Next, we used the above methodology to increase the $c r t S$ gene dose. A crtS expression cassette was constructed by OE-PCR (overlap extension- PCR) and cloned into plasmid pBluescript SK-, resulting in the plasmid pBS-PTEFcrtS-Tact. This cassette was integrated in the DHS3 locus, as its interruption did not cause auxotrophy or greatly affected the carotenogenesis in this yeast. Four DNA fragments (the hygromycin B-resistance selection marker, the crtS gene expression cassettes, and the DHS3 locus "up" and "down" targeting fragments) were individually PCR amplified with primers allowing 50 to $100 \mathrm{bp}$ of overlap between adjacent fragments, and then these fragments were used to co-transform $X$. dendrorhous by electroporation (Figure 3). Four hygromycin B-resistant colonies were obtained. All had the crtS gene expression cassettes at the expected locus, as confirmed by PCR analyses with a comprehensive set of primers. One of these four colonies was randomly selected, named strain Xd_1H1S (DHS3/

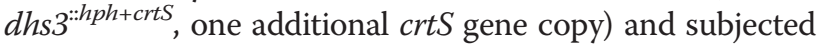
to DRM to result in a homozygous strain, $X d \_2 \mathrm{H} 2 \mathrm{~S}$ $\left(d h s 3^{:: h p h+c r t S} / d h s 3^{:: h p h+c r t S}\right)$. The PCR amplification confirmed the insertion of the hygromycin B resistance and crtS expression cassettes at the DHS3 locus in the heterozygous and homozygous transformants are shown in Figure 4 . 


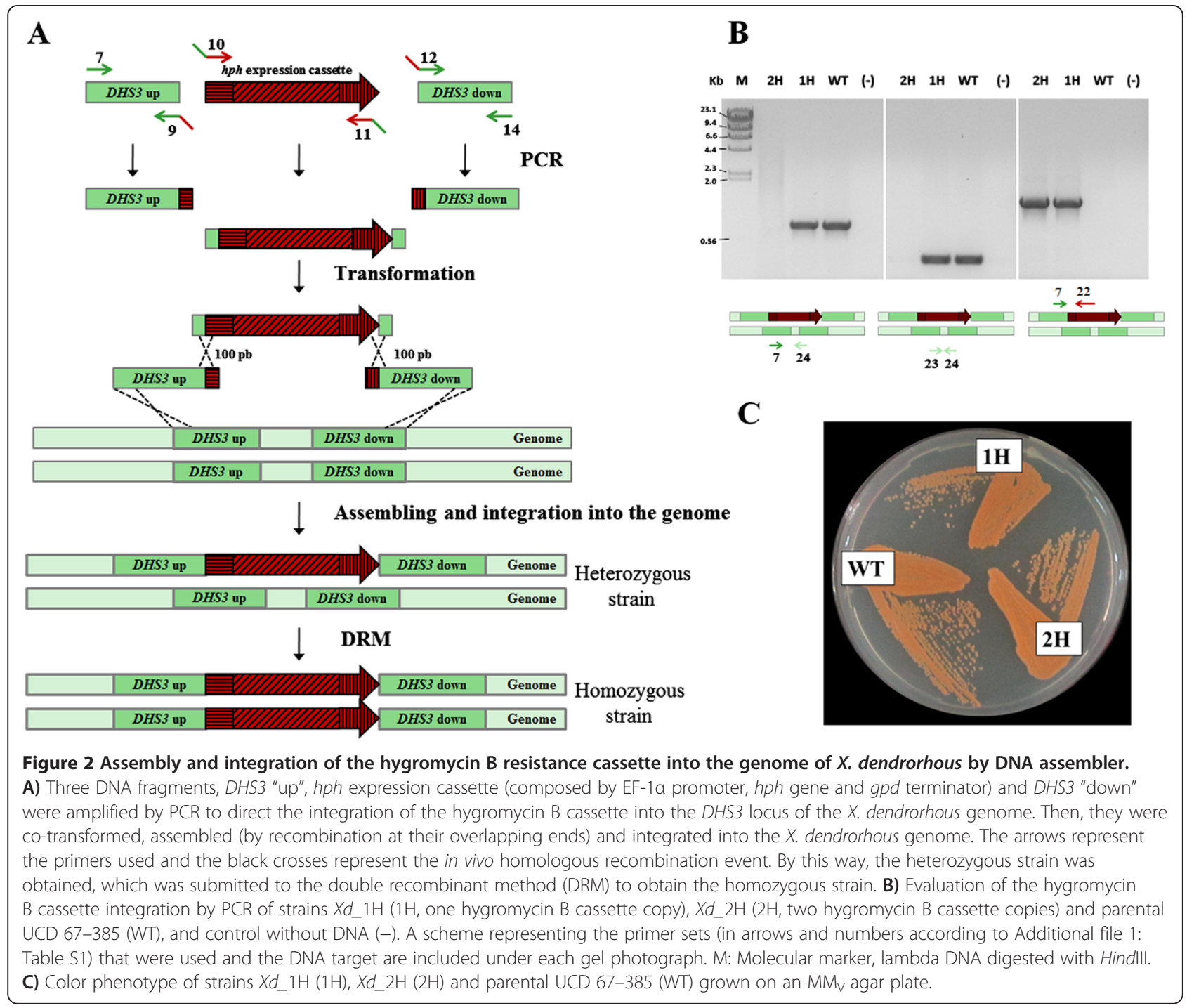

To assess whether the increase in the crtS gene dose effectively leads to an increase in the crtS mRNA levels, RT-qPCR analysis was performed for the wild-type, heterozygous and homozygous transformants grown under the same conditions. Total RNA was extracted after $50 \mathrm{~h}$ of culture (late exponential phase of growth) of each strain as it was observed that the crtS mRNA level reaches its maximum level at this stage [32,33]. The relative crtS expression was normalized to the expression of the actin gene [34], and RT-qPCR analysis revealed that the expression of the crtS gene was increased approximately three fold in the $\mathrm{Xd} \_2 \mathrm{H} 2 \mathrm{~S}$ strain with respect to the wild-type strain (UCD-67-385) at this same growth stage (Figure 5).

\section{Carotenoid production}

To the naked eye, different pigmentation phenotypes among the wild-type, $X d_{-} 1 \mathrm{H} 1 \mathrm{~S}$ and $X d_{-} 2 \mathrm{H} 2 \mathrm{~S}$ strains could be noted. To confirm this observation, the carotenoid content and composition of these strains were analyzed. Total carotenoids were extracted from cell pellets from 24, 50 and 96-h-old yeast cultures (early exponential, late exponential and late stationary phase of growth, respectively) grown at $22^{\circ} \mathrm{C}$ in $\mathrm{YM}$ (yeast malt broth) medium with constant agitation. Carotenoids were extracted, quantified by absorbance at $465 \mathrm{~nm}$ and their composition was analyzed by RP-HPLC. The results are summarized in Table 1. Although the total carotenoid production is not affected in strains that overexpress $\mathrm{crtS}$, it can be observed that after 50 and $95 \mathrm{~h}$ of culture, the percentage of astaxanthin in relation to the other carotenoids is higher in strains expressing additional $c r t S$ gene copies.

\section{Discussion}

There are a limited number of currently available molecular tools to manipulate and transform $X$. dendrorhous. 

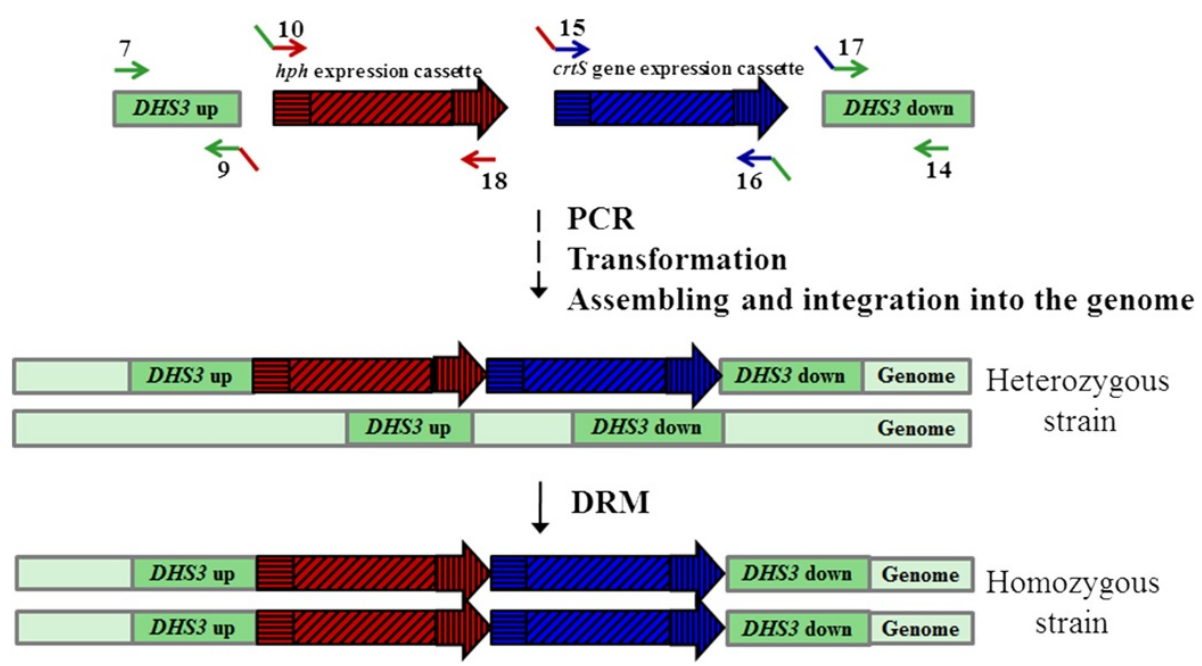

Figure 3 Assembly and integration of hygromycin B resistance and crtS gene expression cassettes into the DHS3 locus of X. dendrorhous by DNA assembler. Four DNA fragments: DHS3 "up", hph expression cassette (composed by EF-1a promoter, hph gene and gpd terminator), crtS gene expression cassette (composed by EF-1a promoter, crtS gene and actin transcription terminator) and DHS3 "down" were amplified by PCR to direct the integration of exogenous genes into the DHS3 locus of the $X$. dendrorhous genome. Arrows represent the primers used (Additional file 1: Table S1).

Until this report, transformation of $X$. dendrorhous usually required the construction of a plasmid for transformation [17,25-27], which involves several conventional steps of sequential cloning such as DNA amplification, endonuclease digestion, in vitro ligation and transformation. If the number of expression cassettes to incorporate in a host is increased, these processes will be time consuming and will depend on the availability of restriction sites in the cloning vector. In contrast, the DNA assembler method requires only DNA fragments with homologous ends. We find that this methodology works in $X$. dendrorhous as we transformed this yeast with three DNA fragments to integrate a hygromycin B resistance cassette into its genome. We also increased the number of crtS gene copies in this yeast by transforming it with four DNA fragments to be assembled (5,098 kb in total) and integrated in the yeast genome. We conclude that this methodology can be employed to engineer $X$. dendrorhous to interrupt or increase gene copy number of several combinations of genes in only one transformation event.

In previous studies attempting to overexpress genes in $X$. dendrorhous, expression cassettes were integrated into ribosomal DNA (rDNA) [17-20]. There are approximately 60 rDNA copies in $X$. dendrorhous [24], which

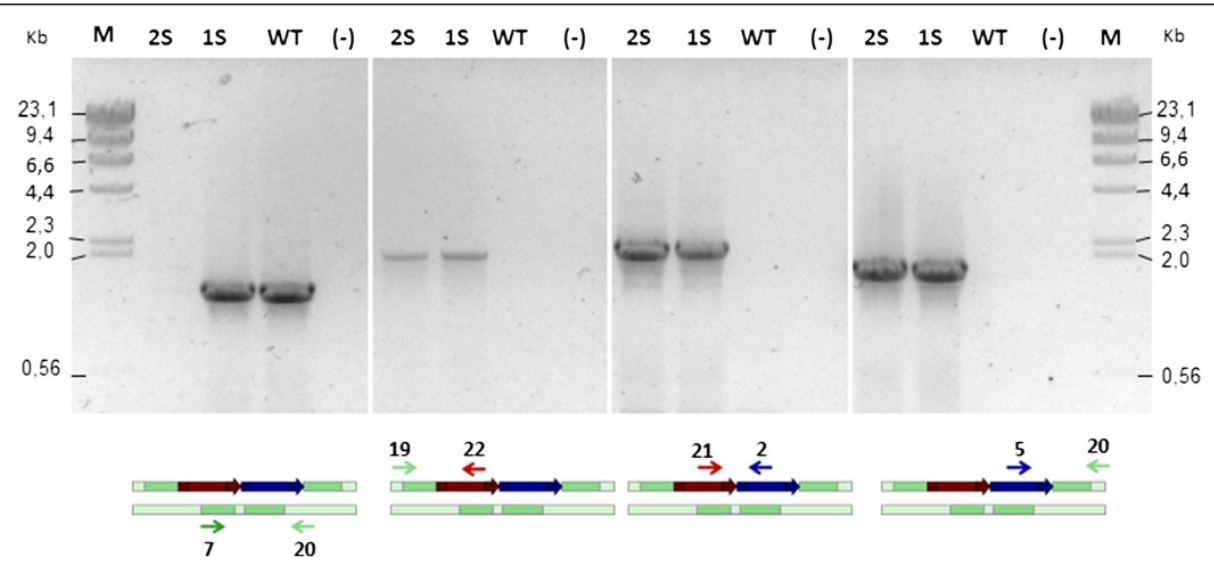

Figure 4 PCR-based analysis of the crtS expression cassette and hygromycin B resistance cassette integration into the genome of $X$. dendrorhous. PCR analyses of Xd_1 H1S (1S, one additional crtS gene copy), Xd_2H2S (2S, two additional crtS gene copies), and parental UCD 67-385 (WT) strains, and the negative control without DNA (-). A scheme representing the primers sets that were used (in arrows and numbers according to Additional file 1: Table S1) and the DNA target are under each gel photograph. The scheme shading is in accordance with Figure 3 . M: Molecular marker, lambda DNA digested with Hindlll. 


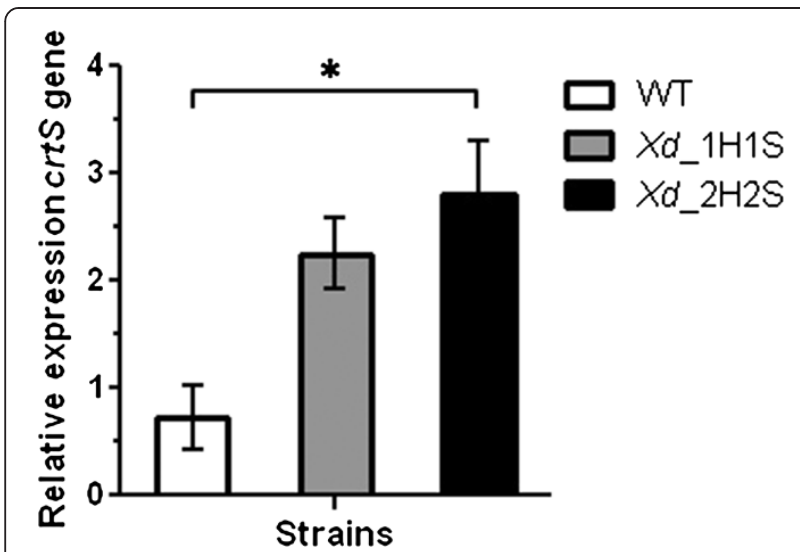

Figure 5 RT-qPCR expression analysis of crtS in the wild-type strain and crtS-overexpressing transformants. The crtS transcript level was normalized to the actin mRNA and was determined from RNA extracted after $50 \mathrm{~h}$ of culture in YM medium from the parental wildtype UCD 67-385 (WT), the heterozygous Xd_1H1S (DHS3/dhs3 $3^{\text {:hph+crts }}$ and homozygous Xd_2H2S (dhs $3^{\text {:hph+crts }} / d$ dhs $3^{\text {:hhh }}$ +crts $)$ strains. Values are the mean \pm standard error of at least three independent cultures. $\left({ }^{*} \mathrm{P} \leq 0.05\right.$; Student's $t$ test $)$

favors multiple integrations of the transformant DNA into the genome. However, due to the tandem repeats in this region, the resulting strains are typically unstable as the integrated genes may be lost by homologous recombination. Additionally, the use of this region makes it difficult to evaluate the effect of gene dose on the production of carotenoids, as it is difficult to quantify the number of integrated cassette copies. For these reasons, we excluded the rDNA region as a target site for integration and focused on integration and resultant target site disruption in a locus that does not strongly modify the yeast physiology. From the analyzed targets, the disruption of locus DHS3 fulfilled this requirement, so it was chosen to integrate the additional $\mathrm{crtS}$ gene copies.

In this work, we studied the effect of altering the metabolic flux between beta-carotene and astaxanthin by increasing the $\mathrm{crtS}$ gene dose. Although the total carotenoid content in strains with one or two additional crtS gene copies was not substantially modified (Table 1), an increased proportion of astaxanthin relative to the total carotenoid content was obtained in the strain with two additional $\mathrm{crtS}$ gene copies compared to the wild-type strain. Astaxanthin represented $96 \%$ versus $68 \%$ of the carotenoids in these strains after $96 \mathrm{~h}$ of culture. In addition, reduced proportions of beta-carotene and intermediate xanthophylls formed during the synthesis of astaxanthin from beta-carotene such as echinenone, hydroxyechinenone, canthaxanthin and phoenicoxanthin. These observations suggest that astaxanthin synthesis is limited by the amount of astaxanthin synthase substrate, beta-carotene. Thus, to achieve a significantly higher level of astaxanthin production, it is also necessary to increase the synthesis of beta-carotene. In agreement with this assumption, it has been observed that the overexpression of the $\operatorname{crtYB}$ gene in $X$. dendrorhous resulted in increased beta-carotene production [17] and recently, the simultaneous overexpression of the $\operatorname{crt} Y B$ and $c r t S$ genes in a $X$. dendrorhous strain created via random mutagenesis that already overproduced astaxanthin resulted in a higher astaxanthin content compared to the parental strain (5,300 ppm) [20]. The methodology described in this work should be a helpful tool to evaluate the consequences of overexpressing different combinations of genes involved in carotenoid production in $X$. dendrorhous.

Although beta-carotene was almost completely exhausted in the transformant strain that overexpressed $c r t S$, phoenicoxanthin, the xanthophyll precursor to astaxanthin, was still present, although its proportion was reduced from $15 \%$ to $6 \%$ after $96 \mathrm{~h}$ of culture in the wild-type and in the $\mathrm{Xd} \_2 \mathrm{H} 2 \mathrm{~S}$ strains, respectively. As astaxanthin synthase is a cytochrome P450 enzyme, it may also be necessary to increase the cytochrome P450 reductase [25] gene dose to enhance the activity of this enzyme.

The DNA assembler methodology that has been effective and successful in $X$. dendrorhous in this study should be useful to overexpress several genes simultaneously to favor the synthesis of carotenoid precursors such as geranylgeranyl pyrophosphate or genes involved in the mevalonate pathway [35] in combination with carotenogenic genes. Thus, using different promoters in the construction of the gene expression cassettes, it should be possible to increase and modulate the production of carotenoids in X. dendrorhous.

\section{Conclusions}

The DNA assembler method is a successful technique to transform $X$. dendrorhous. This technique allowed an increase in the crtS gene copy number in the $X$. dendrorhous genome. The overexpression of this gene did not significantly change the total carotenoid production, but there was an increase in the astaxanthin fraction of carotenoids.

As DNA assembler requires only DNA fragments with homologous ends, this technique could be useful to quickly and easily overexpress several genes simultaneously in $X$. dendrorhous, saving numerous steps involved in conventional cloning methods.

\section{Methods}

The experiments performed in this work were approved by the Facultad de Ciencias - Universidad de Chile, Ethics Committee.

\section{Microorganisms, plasmids, media, and enzymes}

The strains and plasmids that were used and built in this work are listed in Table 2. The wild type $X$. dendrorhous 
Table 1 Carotenoids in wild-type and transformant strains in ppm ( $\mu \mathrm{g}$ per $\mathrm{g}$ of dry yeast)

\begin{tabular}{|c|c|c|c|c|c|c|c|c|c|}
\hline \multirow[b]{4}{*}{ Cultivation time (h) } & \multicolumn{9}{|c|}{$X$. dendrorhous strain [ppm (\%)] } \\
\hline & \multirow{2}{*}{\multicolumn{3}{|c|}{ UCD 67-385 }} & \multirow{2}{*}{\multicolumn{3}{|c|}{$\begin{array}{c}\text { Xd_1H1S } \\
\left(D H S 3 / d h s 3^{:: h p h+c r t S}\right)\end{array}$}} & \multirow{2}{*}{\multicolumn{3}{|c|}{$\begin{array}{c}\text { Xd_2H2S } \\
\left(d h s 3^{:: h p h+c r t S} / d h s 3^{:: h p h+c r t S}\right)\end{array}$}} \\
\hline & & & & & & & & & \\
\hline & 25 & 50 & 96 & 25 & 50 & 96 & 25 & 50 & 96 \\
\hline \multicolumn{10}{|l|}{ Carotenoid: } \\
\hline \multirow[t]{2}{*}{ Total carotenoids } & $\begin{array}{c}22.70 \pm \\
11.16\end{array}$ & $\begin{array}{c}34.31 \pm \\
15.87\end{array}$ & $\begin{array}{c}189.32 \pm \\
86.51\end{array}$ & $\begin{array}{c}14.35 \pm \\
11.71\end{array}$ & $\begin{array}{c}64.21 \pm \\
1.13\end{array}$ & $\begin{array}{c}188.37 \pm \\
6.05\end{array}$ & $\begin{array}{c}12.98 \pm \\
4.93\end{array}$ & $\begin{array}{c}84.79 \pm \\
28.83\end{array}$ & $\begin{array}{c}197.85 \pm \\
34.65\end{array}$ \\
\hline & $(100)$ & $(100)$ & $(100)$ & $(100)$ & $(100)$ & $(100)$ & $(100)$ & $(100)$ & $(100)$ \\
\hline \multirow[t]{2}{*}{ Beta-carotene } & ND & $4.37 \pm 1.25$ & $6.90 \pm 0.57$ & ND & $7.4 \pm 0.01$ & $3.93 \pm 0.08$ & ND & $2.36 \pm 0.29$ & $0.72 \pm 0.22$ \\
\hline & & (13) & $(4)$ & & (12) & (2) & & (3) & (1) \\
\hline \multirow[t]{2}{*}{ Equinenone $^{+}$} & ND & $1.36 \pm 0.31$ & $7.01 \pm 0.46$ & ND & $2.84 \pm 0.01$ & $3.84 \pm 0.05$ & ND & $0.54 \pm 0.32$ & $0.88 \pm 0.03$ \\
\hline & & $(4)$ & (4) & & (4) & (2) & & (1) & (1) \\
\hline \multirow[t]{2}{*}{$\mathrm{OH}$-equinenone $^{+}$} & ND & $4.65 \pm 0.38$ & $11.47 \pm 1.10$ & ND & $5.79 \pm 0.01$ & $7.29 \pm 0.04$ & ND & $8.85 \pm 0.31$ & $1.06 \pm 0.16$ \\
\hline & & (14) & (6) & & (9) & (4) & & (10) & (1) \\
\hline \multirow[t]{2}{*}{ Cantaxanthin $^{\dagger}$} & ND & $0.46 \pm 0.12$ & $2.24 \pm 0.90$ & ND & $0.73 \pm 0$ & $1.41 \pm 0.05$ & ND & $0.39 \pm 0.12$ & $0.83 \pm 0.21$ \\
\hline & & (1) & (1) & & (1) & (1) & & (1) & (1) \\
\hline \multirow[t]{2}{*}{ Phoenicoxanthin $^{\dagger}$} & $0.46 \pm 0.39$ & $3.99 \pm 0.37$ & $28.41 \pm 3.53$ & $0.5 \pm 0.41$ & $6.65 \pm 0.01$ & $15.93 \pm 0.01$ & ND & $5.50 \pm 0.41$ & $11.08 \pm 0.07$ \\
\hline & (2) & (12) & (15) & (3) & (10) & (8) & & (6) & (6) \\
\hline \multirow[t]{2}{*}{ Astaxanthin ${ }^{\dagger}$} & $21.97 \pm 0.62$ & $18.81 \pm 1.77$ & $128.04 \pm 5.06$ & $13.85 \pm 0.41$ & $38.05 \pm 0.04$ & $154.13 \pm 0.09$ & $12.98 \pm 0$ & $65.84 \pm 0.81$ & $179.21 \pm 0.52$ \\
\hline & (97) & (55) & (68) & (97) & (59) & (82) & $(100)$ & (78) & (96) \\
\hline \multirow[t]{2}{*}{ Lycopene } & ND & ND & ND & ND & ND & ND & ND & ND & $0.51 \pm 0.15$ \\
\hline & & & & & & & & & (1) \\
\hline \multirow[t]{2}{*}{ Neurosporene } & ND & $0.10 \pm 0.04$ & ND & ND & $0.54 \pm 0.01$ & ND & ND & ND & ND \\
\hline & & $(0)$ & & & (1) & & & & \\
\hline \multirow[t]{2}{*}{ Torulene } & ND & $0.07 \pm 0.04$ & ND & ND & $0.206 \pm 0.01$ & ND & ND & ND & $0.45 \pm 0.03$ \\
\hline & & (0) & & & (1) & & & & (1) \\
\hline \multirow[t]{2}{*}{ OH-keto-torulene ${ }^{t^{*}}$} & ND & $0.47 \pm 0.20$ & $0.76 \pm 0.52$ & ND & $1.72 \pm 0.02$ & $1.85 \pm 0.02$ & ND & $0.95 \pm 0.24$ & $1.49 \pm 0.26$ \\
\hline & & $(1)$ & $(1)$ & & $(2)$ & $(1)$ & & (1) & $(1)$ \\
\hline
\end{tabular}

ND: Not detected. Table values correspond to the average result from three independent cultures \pm standard deviations. Percentage relative to total carotenoids is indicated in parentheses. ${ }^{+}$: hydroxy or/and keto carotenoid, ${ }^{*}$ : monocyclic carotenoid.

strain UCD 67-385 (ATCC 24230) was used as a parental strain for transformation experiments and $E$. coli DH-5 $\alpha$ was used as a host for plasmid propagation.

$X$. dendrorhous strains were grown at $22^{\circ} \mathrm{C}$ with constant agitation in YM medium (1\% glucose, $0.3 \%$ yeast extract, $0.3 \%$ malt extract and $0.5 \%$ peptone) or minimal $\mathrm{MM}_{\mathrm{V}}+2 \%$ glucose medium [36]. Yeast transformant selection was performed on YM 1.5\% agar plates supplemented with $15 \mu \mathrm{g} / \mathrm{ml}$ hygromycin B. E. coli strains were grown with constant agitation at $37^{\circ} \mathrm{C}$ in LuriaBertani (LB) medium and supplemented with $100 \mu \mathrm{g} / \mathrm{ml}$ ampicillin for plasmid selection and $80 \mu \mathrm{g} / \mathrm{ml} \mathrm{X-gal} \mathrm{(5-}$ bromo-4-chloro-3-indolyl- $\beta$-D-galactopyranoside) for recombinant clone selection [37].

Enzymes were purchased from Promega (Taq DNA pol, restriction enzymes, M-MLV reverse transcriptase) and Pfu DNA pol was purchased from Agilent Technologies.

\section{DNA amplification}

All of the nucleotides used in this work were purchased from Alpha DNA (Montreal, Canada) or from Integrated DNA Technologies and are listed in Additional file 1: Table S1. PCR reactions were performed in a final volume of $25 \mu \mathrm{l}$ containing $2 \mathrm{U}$ of Taq DNA pol, $2.5 \mu \mathrm{l}$ of 10× Taq buffer, $0.5 \mu \mathrm{l}$ of $10 \mathrm{mM}$ dNTPs, $1 \mu \mathrm{l}$ of $50 \mathrm{mM}$ $\mathrm{MgCl}_{2}, 1 \mu \mathrm{l}$ of $25 \mu \mathrm{M}$ of each primer and 10-20 ng of template DNA. Amplification was performed in a DNA thermal cycler 2720 (Applied Biosystems) as follows: initial denaturation at $95^{\circ} \mathrm{C}$ for $3 \mathrm{~min}$; 35 cycles of denaturation at $94^{\circ} \mathrm{C}$ for $30 \mathrm{~s}$, annealing at $55^{\circ} \mathrm{C}$ for $30 \mathrm{~s}$, synthesis at $72^{\circ} \mathrm{C}$ for $3 \mathrm{~min}$ and a final extension step at $72^{\circ} \mathrm{C}$ for $10 \mathrm{~min}$. Samples were kept at $4^{\circ} \mathrm{C}$ until use. The amplicons were separated by $0.8 \%$ agarose gel electrophoresis in TAE buffer containing $0.5 \mu \mathrm{g} / \mathrm{ml}$ ethidium bromide [37] followed by DNA purification using the 
Table 2 Strains and plasmids used and built in this work

\begin{tabular}{|c|c|c|}
\hline & Genotype or relevant features & $\begin{array}{l}\text { Source or } \\
\text { reference }\end{array}$ \\
\hline \multicolumn{3}{|l|}{ Strains: } \\
\hline \multicolumn{3}{|l|}{ E. coli: } \\
\hline $\mathrm{DH}-5 \mathrm{a}$ & F- $\varphi 80 d$ lacZ $\Delta$ M15 (lacZY-argF) U169 deoR recA1 endA1 hsdR17(rk- mk+) phoA supE44l- thi-1 gyrA96 relA1 & [37] \\
\hline \multicolumn{3}{|l|}{ X. dendrorhous: } \\
\hline UCD 67-385 & ATCC 24230, wild-type. Diploid strain [31]. & ATCC \\
\hline$X d_{-} 1 \mathrm{H}$ & $\begin{array}{l}\text { (DHS3/dhs3: }{ }^{\text {hph }} \text { ). Heterozygote transformant derived from UCD 67-385 containing an allele of the DHS3 locus with } \\
\text { a deletion and a hygromycin B resistance cassette. }\end{array}$ & This work \\
\hline $\mathrm{Xd} \_2 \mathrm{H}$ & $\begin{array}{l}\left(\text { dhs } 3^{: \text {:hph }} / d h s 3^{: \text {:hph }}\right) \text {. Homozygote transformant derived from Xd_1H by DRM [30] with a deletion and a hygromycin } \\
\text { B resistance cassette in both alleles of the DHS3 locus. }\end{array}$ & This work \\
\hline$X d_{-} 1 \mathrm{H} 1 \mathrm{~S}$ & $\begin{array}{l}\text { (DHS3/dhs } 3^{\text {:hph+crtS }} \text { ). Heterozygote transformant derived from UCD } 67-385 \text { with a deletion in one DHS3 allele and } \\
\text { bearing the hygromycin B resistance cassette and the crtS gene expression cassette. }\end{array}$ & This work \\
\hline$X d \_2 H 2 S$ & $\begin{array}{l}\left(d h s 3^{: h p h+c r t s} / d h s 3^{: h p h+c r t S}\right) \text {. Homozygote transformant derived from Xd_1H1S by DRM with a deletion in both DHS3 } \\
\text { alleles and bearing the hygromycin B resistance cassette and the crtS gene expression cassette. }\end{array}$ & This work \\
\hline \multicolumn{3}{|l|}{ Plasmids: } \\
\hline $\begin{array}{l}\text { pBluescript } \\
\text { SK- (pBS) }\end{array}$ & ColE1 ori; AmpR; cloning vector for blue-white selection & Stratagene \\
\hline pMN-hph & $\begin{array}{l}\text { pBS containing a cassette of } 1.8 \mathrm{~kb} \text { bearing the resistance Hygromycin B (hph) gene under EF-1 a promoter and } \\
\text { gpd transcription terminator of } X \text {. dendrorhous in its EcoRV site. }\end{array}$ & [30] \\
\hline pXd_Ex_crtS & pBS containing the cDNA encoding the $X$. dendrorhous crtS gene in its EcoRV site. & [26] \\
\hline $\begin{array}{l}\text { pBS-PTEF-crtS- } \\
\text { Tact }\end{array}$ & $\begin{array}{l}\text { pBS containing a 2,367 bp cassette bearing the cDNA encoding the } X \text {. dendrorhous crtS gene under EF-1a } \\
\text { promoter and the actin transcription terminator from } X \text {. dendrorhous in the EcoRV site. }\end{array}$ & This work \\
\hline
\end{tabular}

ATCC: American Type Culture Collection.

DHS3 [GenBank: JN849376.1]: locus downstream of the X. dendrorhous HIS3 gene [GenBank: JN849374.1].

Glassmilk method [38] for sequencing or gene expression cassette construction.

To reduce the error rate in DNA amplification for Overlap Extension-PCR (OE-PCR, [39] and DNA fragment amplification for $X$. dendrorhous transformation, $P f u$ DNA pol was used instead of Taq DNA pol following the manufacturer's instructions.

\section{Cloning and construction of the crtS gene expression cassette}

The $c r t S$ gene expression cassette was constructed by Overlap Extension-PCR (OE-PCR) [39] containing 1,674 bp of the cDNA of the crtS gene [GenBank: DQ002007.1] under the control of $393 \mathrm{bp}$ of the $X$. dendrorhous EF- $1 \alpha$ promoter [26] and $300 \mathrm{bp}$ of the actin transcription terminator of [GenBank: X89898.1]. The EF-1 $\alpha$ promoter region was amplified by PCR with primers 1 and 2 (Additional file 1 : Table S1) using pMN-hph as a template (Table 2), and the actin gene terminator was amplified from UCD 67-385 genomic DNA using primers 5 and 6 (Additional file 1: Table S1). The $c r t S$ cDNA was amplified from $\mathrm{pXd}$ _Ex_crtS (Table 2) with primers 3 and 4 (Additional file 1: Table S1). First, the promoter region was joined to the crtS cDNA and then the hybrid product was joined to the actin gene transcription terminator by OE-PCR. The resulting cassette was cloned into the EcoRV site of plasmid pBS (pBS-PTEFcrtS-Tact, Table 2) and was sequenced on both strands using a DYEnamic ET Terminator Kit (Amersham Bioscience) in an ABI 3100 Avant genetic analyzer. DNA sequences were analyzed with Vector NTI Suite 10 (Informax) and bioinformatics programs available online.

\section{$X$. dendrorhous transformation}

$X$. dendrorhous transformation was performed by electroporation according to [40] and [41]. Electrocompetent cells were prepared from an exponential culture with $\mathrm{DO}_{600 \mathrm{~nm}}=1.2$, cultured in YM medium and electroporated using a BioRad Gene Pulser Xcell with PC and CE modules under the following conditions: $125 \mathrm{mF}, 600 \Omega$, $0.45 \mathrm{kV}$. The yeast were transformed with $4 \mu \mathrm{l}$ of a mixture of purified DNA fragments ( $1 \mu \mathrm{g}$ of each fragment) that were amplified by PCR with Pfu DNA pol and primers with $5^{\prime}$ complementary ends to allow their assembly by homologous recombination. In each transformation event, in addition to the selection marker and/or the crtS gene expression cassette, "up" and "down" DNA fragments were included targeting the insertion into the integration site. The hygromycin B resistance and crtS gene expression cassettes were amplified from plasmids pMNHyg and pBS-PTEF-crtS-Tact, respectively, and the "up" and "down" DNA fragments were amplified from $X$. dendrorhous wild-type genomic DNA. Transformant selection was performed on YM $1.5 \%$ agar plates supplemented with $15 \mu \mathrm{g} / \mathrm{ml}$ hygromycin B. As strain 
UCD 67-385 is diploid [31], heterozygous transformants were obtained. To obtain homozygous transformants, the double recombinant method (DRM) [30] was applied. The transformant strains were confirmed as $X$. dendrorhous by examining the ITS1, 5.8 rRNA gene and ITS2 DNA sequences [42,43].

\section{RNA extraction, single strand DNA synthesis and RT-qPCR}

To measure the relative crtS gene expression, total RNA was extracted from $50 \mathrm{~h}$ yeast cultures (late exponential phase of growth) grown at $22^{\circ} \mathrm{C}$ with constant agitation in YM medium. The cell pellets from $5 \mathrm{ml}$ culture aliquots were frozen with liquid nitrogen and stored at $-80^{\circ} \mathrm{C}$ until use. Total RNA extraction from the cell pellets was performed by mechanical rupture with $0.5 \mathrm{~mm}$ glass beads (BioSpec) during $10 \mathrm{~min}$ of vortexing, followed by the addition of Tri-Reagent (Ambion). The lysate was incubated for $5 \mathrm{~min}$ at room temperature and $200 \mu \mathrm{l}$ of chloroform per $\mathrm{ml}$ of Tri-Reagent used was added, mixed, and centrifuged for $5 \mathrm{~min}$ at 4,000 $\times \mathrm{g}$. Following this centrifugation, the aqueous phase was recovered. The RNA was precipitated by adding two volumes of isopropanol and incubating at room temperature for $10 \mathrm{~min}$. The RNA was washed with $75 \%$ ethanol, suspended in RNase-free $\mathrm{H}_{2} \mathrm{O}$ and quantified by absorbance determination at $260 \mathrm{~nm}$ in a double beam Shimadzu UV-150-20 spectrophotometer.

The synthesis of cDNA was performed according to the M-MLV reverse transcriptase (Invitrogen) manufacturer's protocol with $5 \mu \mathrm{g}$ of total RNA in a final volume of $11 \mu$ l. The determination of the relative $\operatorname{crtS}$ gene expression levels was performed in an Mx3000P quantitative PCR system (Stratagene) using $1 \mu \mathrm{l}$ of the reverse transcription reaction, $0.25 \mu \mathrm{M}$ of each primer (Additional file 1: Table S1) and $10 \mu \mathrm{l}$ of the SensiMix SYBR Green I (Quantace) kit reagent in a final volume of $20 \mu \mathrm{l}$. The obtained $\mathrm{Ct}$ values were normalized to the respective value of the actin gene [GenBank: X89898.1] [44] and expressed using the $2_{T}^{-\Delta C}$ method $[45,46]$.

\section{Carotenoid extraction and RP-HPLC}

Carotenoids were extracted from cell pellets from 24, 50 and 96-hours-old yeast cultures (early exponential, late exponential and late stationary phase of growth, respectively) grown at $22^{\circ} \mathrm{C}$ with constant agitation in YM medium using the acetone extraction method [12]. Total carotenoids were quantified by absorbance at $465 \mathrm{~nm}$ using an absorption coefficient of $\mathrm{A}_{1 \%}=2,100$. The analyses were performed at least in triplicate, and pigments were normalized relative to the dry weight of the yeast. Carotenoids were separated by RP-HPLC using a reverse phase RP-18 LiChroCART 125-4 (Merck) column with acetonitrile:methanol:isopropanol $(85: 10: 5 \mathrm{v} / \mathrm{v})$ as the mobile phase under isocratic conditions with a $1 \mathrm{ml} / \mathrm{min}$ flux. The elusion spectra were recovered using a diode array detector, and carotenoids were identified by their spectra and retention time according to standards.

\section{Additional file}

Additional file 1: Table S1. Primers used in this work.

\section{Competing interests}

The authors declare that they no competing interests.

\section{Authors' contributions}

GC constructed the crtS expression module, performed the $X$. dendrorhous transformations and transformant analyses, carotenoid analyses and gene expression analyses. SB contributed to the RT-qPCR gene expression analyses. MCR and MB contributed to the study design and results analyses. VC participated in the experimental design and coordination. JA conceived the study and participated in its design and coordination. JA, MB, VC drafted the manuscript. All authors read and approved the final manuscript.

\section{Acknowledgements}

This work was supported by projects: Fondecyt 11121200 to JA and Fondecyt 1100324 to VC.

\section{Author details}

'Departamento de Ciencias Ecológicas, Facultad de Ciencias, Universidad de Chile, Las Palmeras 3425, Casilla, Santiago 653, Chile. 'Departamento de Química, Facultad de Ciencias, Universidad de Chile, Las Palmeras 3425, Casilla, Santiago 653, Chile.

Received: 12 July 2013 Accepted: 4 October 2013

Published: 9 October 2013

\section{References}

1. Britton G, Pfander H: Liaaen-Jensen S. Handbook. Birkhäuser Verlag: Carotenoid; 1998.

2. Schroeder WA, Johnson EA: Carotenoids protect Phaffia rhodozyma against singlet oxygen damage. J Indust Microbiol 1995, 14:502-507.

3. Miki W: Biological functions and activities of animal carotenoids. Pure App/ Chem 1991, 63:141-146.

4. Higuera-Ciapara I, Felix-Valenzuela L, Goycoolea FM: Astaxanthin: a review of its chemistry and applications. Crit Rev Food Sci Nutr 2006, 46:185-196.

5. Hu Z-C, Zheng Y-G, Wang Z, Shen Y-C: Effect of sugar-feeding strategies on astaxanthin production by Xanthophyllomyces dendrorhous. World J Microb Biot 2005, 21:771-775

6. Yamane $Y$, Higashida $K$, Nakashimada $Y$, Kakizono $T$, Nishio N: Influence of oxygen and glucose on primary metabolism and astaxanthin production by Phaffia rhodozyma in batch and fed-batch cultures: kinetic and stoichiometric analysis. Appl Environ Microbiol 1997, 63:4471-4478.

7. Liu YS, Wu JY: Use of n-hexadecane as an oxygen vector to improve Phaffia rhodozyma growth and carotenoid production in shake-flask cultures. J Appl Microbiol 2006, 101:1033-1038.

8. Johnson EA, Lewis MJ: Astaxanthin formation by the yeast Phaffia rhodozyma. J Gen Microbiol 1979, 115:173-183.

9. Hu Z-C, Zheng Y-G, Wang Z, Shen Y-C: pH control strategy in astaxanthin fermentation bioprocess by Xanthophyllomyces dendrorhous. Enzyme Microb Technol 2006, 39:586-590.

10. Flores-Cotera L, Martin R, Sanchez S: Citrate, a possible precursor of astaxanthin in Phaffia rhodozyma: influence of varying levels of ammonium, phosphate and citrate in a chemically defined medium. Appl Microbiol Biotechnol 2001, 55:341-347.

11. An G-H, Johnson EA: Influence of light on growth and pigmentation of the yeast Phaffia rhodozyma. Antonie Van Leeuwenhoek 1990, 57:191-203.

12. An G-H, Schuman DB, Johnson EA: Isolation of Phaffia rhodozyma mutants with increased astaxanthin content. Appl Environ Microbiol 1989, 55:116-124.

13. Lewis MJ, Ragot N, Berlant MC, Miranda M: Selection of astaxanthinoverproducing mutants of Phaffia rhodozyma with $\beta$-ionone. Appl Environ Microbiol 1990, 56:2944-2945. 
14. Retamales $P$, León $R$, Martinez C, Hermosilla G, Pincheira G, Cifuentes V: Complementation analysis with new genetic markers in Phaffia rhodozyma. Antonie Van Leeuwenhoek 1998, 73:229-236.

15. Ukibe K, Katsuragi T, Tani Y, Takagi H: Efficient screening for astaxanthinoverproducing mutants of the yeast Xanthophyllomyces dendrorhous by flow cytometry. FEMS Microbiol Lett 2008, 286:241-248.

16. Schmidt I, Schewe H, Gassel S, Jin C, Buckingham J, Hümbelin M, Sandmann G, Schrader J: Biotechnological production of astaxanthin with Phaffia rhodozyma/Xanthophyllomyces dendrorhous. Appl Microbiol Biotechnol 2011, 89:555-571.

17. Verdoes JC, Sandmann G, Visser H, Diaz M, van Mossel M, van Ooyen AJJ: Metabolic engineering of the carotenoid biosynthetic pathway in the yeast Xanthophyllomyces dendrorhous (Phaffia rhodozyma). Appl Environ Microbiol 2003, 69:3728-3738.

18. Visser H, Sandmann G, Verdoes JC: Xanthophylls in Fungi. In Microbial Processes and Products. Volume 18. Edited by Barredo JL. New York: Humana Press; 2005:257-272.

19. Breitenbach J, Visser H, Verdoes JC, van Ooyen AJJ, Sandmann G: Engineering of geranylgeranyl pyrophosphate synthase levels and physiological conditions for enhanced carotenoid and astaxanthin synthesis in Xanthophyllomyces dendrorhous. Biotechnol Lett 2011, 33:755-761

20. Gassel S, Schewe H, Schmidt I, Schrader J, Sandmann G: Multiple improvement of astaxanthin biosynthesis in Xanthophyllomyces dendrorhous by a combination of conventional mutagenesis and metabolic pathway engineering. Biotechnol Lett 2013, 35:565-569.

21. Andrewes AG, Phaff HJ, Starr MP: Carotenoids of Phaffia rhodozyma, a redpigmented fermenting yeast. Phytochemistry 1976, 15:1003-1007.

22. An G-H, Cho M-H, Johnson EA: Monocyclic carotenoid biosynthetic pathway in the yeast Phaffia rhodozyma (Xanthophyllomyces dendrorhous). J Biosci Bioeng 1999, 88:189-193.

23. Shao Z, Zhao $H$, Zhao $H$ : DNA assembler, an in vivo genetic method for rapid construction of biochemical pathways. Nucleic Acids Res 2009, 37:e16.

24. Wery J, Gutker D, Renniers ACHM, Verdoes JC, van Ooyen AJJ: High copy number integration into the ribosomal DNA of the yeast Phaffia rhodozyma. Gene 1997, 184:89-97.

25. Alcaíno J, Barahona S, Carmona M, Lozano C, Marcoleta A, Niklitschek M, Sepúlveda D, Baeza M, Cifuentes V: Cloning of the cytochrome p450 reductase $(c r t R)$ gene and its involvement in the astaxanthin biosynthesis of Xanthophyllomyces dendrorhous. BMC Microbiol 2008 8:169.

26. Niklitschek M, Alcaíno J, Barahona S, Sepúlveda D, Lozano C, Carmona M, Marcoleta A, Martínez C, Lodato P, Baeza M: Genomic organization of the structural genes controlling the astaxanthin biosynthesis pathway of Xanthophyllomyces dendrorhous. Biol Res 2008, 41:93-108.

27. Loto I, Gutiérrez MS, Barahona S, Sepúlveda D, Martínez-Moya P, Baeza M, Cifuentes $V$, Alcaíno J: Enhancement of carotenoid production by disrupting the C22-sterol desaturase gene (CYP61) in Xanthophyllomyces dendrorhous. BMC Microbiol 2012, 12:235.

28. Álvarez V, Rodríguez-Sáiz M, de la Fuente JL, Gudiña EJ, Godio RP, Martín JF, Barredo JL: The crtS gene of Xanthophyllomyces dendrorhous encodes a novel cytochrome-P450 hydroxylase involved in the conversion of $\beta$ carotene into astaxanthin and other xanthophylls. Fungal Genet Biol 2006, 43:261-272

29. Ojima K, Breitenbach J, Visser H, Setoguchi Y, Tabata K, Hoshino T, van den Berg J, Sandmann G: Cloning of the astaxanthin synthase gene from Xanthophyllomyces dendrorhous (Phaffia rhodozyma) and its assignment as a $\beta$-carotene 3-hydroxylase/4-ketolase. Mol Genet Genomics 2006, 275:148-158.

30. Niklitschek M, Baeza M, Fernández-Lobato M, Cifuentes V: Generation of Astaxanthin Mutants in Xanthophyllomyces dendrorhous Using a Double Recombination Method Based on Hygromycin Resistance. In Microbial Carotenoids From Fungi, Volume 898. Edited by Barredo JL. New York: Humana Press; 2012:219-234

31. Hermosilla G, Martínez C, Retamales P, León R, Cifuentes V: Genetic determination of ploidy level in Xanthophyllomyces dendrorhous. Antonie Van Leeuwenhoek 2003, 84:279-287.

32. Lodato P, Alcaíno J, Barahona S, Niklitschek M, Carmona M, Wozniak A Baeza M, Jiménez A, Cifuentes V: Expression of the carotenoid biosynthesis genes in Xanthophyllomyces dendrorhous. Biol Res 2007, 40:73.

33. Wozniak A, Lozano C, Barahona S, Niklitschek M, Marcoleta A, Alcaíno J, Sepulveda D, Baeza M, Cifuentes V: Differential carotenoid production and gene expression in Xanthophyllomyces dendrorhous grown in a nonfermentable carbon source. FEMS Yeast Res 2011, 11:252-262.

34. Radonić A, Thulke S, Mackay IM, Landt O, Siegert W, Nitsche A: Guideline to reference gene selection for quantitative real-time PCR. Biochem Biophys Res Commun 2004, 313:856-862.

35. Miziorko HM: Enzymes of the mevalonate pathway of isoprenoid biosynthesis. Arch Biochem Biophys 2011, 505:131-143.

36. Retamales P, Hermosilla G, Leon R, Martínez C, Jimenez A, Cifuentes V: Development of the sexual reproductive cycle of Xanthophyllomyces dendrorhous. J Microbiol Meth 2002, 48:87-93.

37. Sambrook J, Russell DW: Molecular cloning: a laboratory manual. Cold Spring Harbour, New York: Cold Spring Harbour Laboratory Press; 2001. Volume 1-3.

38. Boyle JS, Lew AM: An inexpensive alternative to glassmilk for DNA purification. Trends Genet 1995, 11:8.

39. Horton RM, Hunt HD, Ho SN, Pullen JK, Pease LR: Engineering hybrid genes without the use of restriction enzymes: gene splicing by overlap extension. Gene 1989, 77:61-68.

40. Adrio $\mathrm{J}$, Veiga M: Transformation of the astaxanthin-producing yeast Phaffia rhodozyma. Biotechnol Tech 1995, 9:509-512.

41. Kim IG, Nam SK, Sohn JH, Rhee SK, An GH, Lee SH, Choi ES: Cloning of the ribosomal protein L41 gene of Phaffia rhodozyma and its use as a drug resistance marker for transformation. Appl Environ Microbiol 1998, 64:1947-1949

42. Fell JW, Blatt GM: Separation of strains of the yeasts Xanthophyllomyces dendrorhous and Phaffia rhodozyma based on rDNA IGS and ITS sequence analysis. J Ind Microbiol Biotechnol 1999, 23:677-681.

43. Reyes E, Barahona S, Fischman O, Niklitschek M, Baeza M, Cifuentes V: Genetic polymorphism of clinical and environmental strains of Pichia anomala. Biol Res 2004, 37:747-757.

44. Wery J, Dalderup MJM, Ter Linde J, Boekhout T, Van Ooyen AJJ: Structural and phylogenetic analysis of the actin gene from the yeast Phaffia rhodozyma. Yeast 1996, 12:641-651.

45. Livak KJ, Schmittgen TD: Analysis of relative gene expression data using real-time quantitative PCR and the 2(-Delta Delta $C(T)$ ) Method. Methods 2001, 25:402-408.

46. Schmittgen TD, Livak KJ: Analyzing real-time PCR data by the comparative CT method. Nat Protoc 2008, 3:1101-1108.

doi:10.1186/1472-6750-13-84

Cite this article as: Contreras et al:: Increase in the astaxanthin synthase gene (crtS) dose by in vivo DNA fragment assembly in

Xanthophyllomyces dendrorhous. BMC Biotechnology 2013 13:84.

\section{Submit your next manuscript to BioMed Central and take full advantage of:}

- Convenient online submission

- Thorough peer review

- No space constraints or color figure charges

- Immediate publication on acceptance

- Inclusion in PubMed, CAS, Scopus and Google Scholar

- Research which is freely available for redistribution 